\title{
SOME PROBLEMS IN THE ENUMERATION \\ OF LABELLED GRAPHS
}

\author{
NICHOLAS C. WORMALD
}

A method of counting labelled 3-connected graphs is presented. The enumeration is accomplished by employing a new characterisation of 3-connected graphs, based on an examination of the graphs which may result when a line is removed from a 3-connected graph. The same general principle is first applied to counting connected and 2-connected labelled graphs. In all three cases, it is necessary to keep track of both the numbers of points and of lines. Numerical results are supplied, showing the numbers of connected, 2-connected and 3-connected labelled graphs with $p$ points for $p$ up to 20 .

The characterisation result for 3-connected graphs also allows us to count labelled 3-connected cubic graphs. Labelled cubic graphs and labelled connected cubic graphs have already been counted by Read. As no cubic graph is 4-connected, the picture is completed by counting labelled 2-connected cubic graphs. This is done by taking into consideration graphs which are cubic except for one or two points of degree 2 . The same method is used to re-derive some of Read's results for labelled cubic graphs and labelled connected cubic graphs. Numerical results are listed for graphs with up to 30 points.

An asymptotic formula for the number of labelled cubic graphs with a given number of points was also found by Read. A generalisation of this problem is solved here by beginning with a known result concerning the asymptotic enumeration of matrices with given row and column sums. By this

Received 7 November 1979. Thesis submitted to the University of Newcastle, December 1978. Degree approved, June 1979. Supervisor: Professor R.W. Robinson. 
means we find an asymptotic formula for the number of labelled graphs (or pseudographs) with points of given degrees, as long as the degrees of the points in the graphs (pseudographs) are bounded. The numbers of cycles of given fixed length can also be specified. This yields, for example, an asymptotic formula for the number of labelled r-regular graphs with girth $j$, for fixed $r$ and $j$.

The asymptotic formula for the number of labelled graphs with points of given degrees is used to show that almost all labelled graphs with points of given degrees lying between $r$ and $R$ are $r$-connected, where $r$ and $R$ are fixed, $3 \leq r \leq R$. This implies, for instance, that almost all labelled $r$-regular graphs are $r$-connected for any fixed $r \geq 3$. A similar procedure is used to show that for fixed $k>0$ and $r \geq 3$, almost all labelled $r$-regular graphs with girth at least $[k /(r-2)\rceil$ are cyclically $k$-connected. This provides an asymptotic formula for the number of labelled cyclically $k$-connected $r$-regular graphs with $p$ points, for fixed $k$ and $r$. 